\title{
Health- or Environment-Focused Text Messages as a Potential Strategy to Increase Plant-Based Eating among Young Adults: An Exploratory Study
}

\author{
Tze Joo Lim ${ }^{1}$, Richard Nii Okine ${ }^{2}$ and Jonathan C. Kershaw ${ }^{1, * D}$ \\ 1 Department of Public and Allied Health, Bowling Green State University, Bowling Green, OH 43403, USA; \\ tlim@bgsu.edu \\ 2 Department of Mathematics and Statistics, Bowling Green State University, Bowling Green, OH 43403, USA; \\ rnokine@bgsu.edu \\ * Correspondence: jkersha@bgsu.edu; Tel.: +1-419-372-4579
}

Citation: Lim, T.J.; Okine, R.N.; Kershaw, J.C. Health- or

Environment-Focused Text Messages as a Potential Strategy to Increase Plant-Based Eating among Young Adults: An Exploratory Study. Foods 2021, 10, 3147. https://doi.org/

$10.3390 /$ foods 10123147

Academic Editor: Giancarlo Statti

Received: 24 November 2021

Accepted: 16 December 2021

Published: 19 December 202

Publisher's Note: MDPI stays neutral with regard to jurisdictional claims in published maps and institutional affiliations.

Copyright: (C) 2021 by the authors Licensee MDPI, Basel, Switzerland. This article is an open access article distributed under the terms and conditions of the Creative Commons Attribution (CC BY) license (https:// creativecommons.org/licenses/by/ $4.0 /)$

\begin{abstract}
Previous plant-based diet (PBD) adoption strategies have primarily focused on health rather than environmental rationale and meat reduction rather than plant-based protein promotion. In this study, we explored the effect of a theory-informed text-message intervention on dietary intentions and behaviors in young adult omnivores and the potential explanatory role of PBD beliefs, subjective norm, self-efficacy, moral norm, and health and environmental values. Participants completed baseline questionnaires and reported dietary intake before being randomly assigned to receive 2-3 health- or environment-focused text messages per week for eight weeks and then repeated baseline assessments. Although we did not see significant changes in meat or plant protein intake, we did observe a marked decrease in intentions to consume animal protein and a marginal increase in fruit and vegetable consumption intention. We identified subjective norms, self-efficacy, and moral satisfaction as the strongest predictors of changes in intention to consume animal or plant protein. Although few group differences were observed, those receiving environment-focused text messages experienced a greater change in values and were more likely to increase vegetable intake. Messages that improve sustainability awareness and provide practical adoption strategies may be part of an effective strategy to influence PBD intake among young adults.
\end{abstract}

Keywords: text message; plant-based diet; meat consumption; sustainability; moral satisfaction; self-efficacy; subjective norm; protein intake

\section{Introduction}

Adoption of plant-based diets (PBD) improves both health and environmental outcomes and aligns with recommendations stated in the Dietary Guidelines for Americans (DGA) [1,2]. PBD emphasize vegetables, fruits, whole grains, nuts, seeds, and their manufactured products and generally focus on reduction rather than elimination of food items from animal sources. However, for several decades, approximately two-thirds of American protein intake has come from animal foods [3-6]. At the same time, many populations continue to fall short of dietary targets despite introduction of the DGA in 1980 [1,2,7,8]. This relatively static state of diet quality suggests that health-focused strategies alone may be insufficient to stimulate meaningful changes. Considering the rising interest in environmental sustainability $[9,10]$, environment-focused messaging may represent a novel strategy to complement existing efforts to improve diet quality.

\subsection{Health and Environmental Effects of Plant-Based Diets}

Plant foods are health-promoting and protective against some diseases [11,12]. Previous studies have consistently shown that a plant-based diet reduces the risk of cardiovascular disease-related mortality, most chronic diseases, type 2 diabetes, hypertension, some 
cancers, and obesity [11,12]. Plant foods also provide the only source of phytochemicals and fibers, which play a role in health promotion and disease prevention [12]. While animal foods are rich in essential nutrients, such as iron, zinc, vitamin D, calcium, and essential fatty acids [12], certain animal products may increase disease risk if consumed in excess. For example, some animal products contain high amounts of saturated fats and other compounds that have putative roles in progression of heart disease, cancer, and other diseases [12,13].

In addition to health benefits, PBD contribute to a number of environmental benefits. Non-vegetarian diets require 2.5 times more energy, 2.9 times more water, 13 times more fertilizer, and 1.4 times more pesticides than vegetarian diets [14]. The 2015 U.S. Dietary Guidelines Advisory Committee concluded that a diet higher in plant-based foods (whole grains, legumes, nuts, seeds, fruits, and vegetables) and lower in animal-based foods is better for health and the environment than the typical U.S. diet $[15,16]$.

\subsection{Facilitators and Barriers to Adoption of Plant-Based Diets}

Although plant-based diets bring about many health and sustainability benefits, shifting dietary patterns is complex. Consumers' attitudes toward and intake of PBD vary widely and are associated with differences in gender, income, age, race/ethnicity, and political leaning $[17,18]$. Consumers who have a positive attitude toward prosumerism, ethics, health, and naturalness are supportive of a transition to a plant-based diet, while social image and pleasure are barriers to a transition to a plant-based diet [19]. Consumers whose established diets consist of beans and soy products have a high preference for plant-based food due to health, weight maintenance, and natural concerns compared with those with high intakes of animal food [20]. Interestingly, those who are undergoing a dietary transition toward the consumption of plant proteins endorsed higher regard for health, natural concerns, price, sociability, and social image [20].

While interest in sustainable eating is growing, general confusion regarding its meaning and a lack of awareness of the need to consume more plant-based foods limit its more widespread adoption. For example, while most Americans have heard of PBD, many people associate sustainability with only tangentially related factors, such as organic or non-genetically engineered foods $[10,21]$. Furthermore, several knowledge-related (e.g., unaware of the need to consume PBD) and ability / access-related barriers to PBD consumption (e.g., cost, knowledge regarding what to buy or how to cook, and perceived effort to prepare) limit its adoption [22]. Thus, addressing the gap in awareness and abilities may encourage a shift to PBD.

\subsection{Text-Message Intervention as a Tool for Nutrition/Health Behavior Change}

Due to the widespread ownership of mobile phones across gender, age, race, education level, income level, and geographical location [23], short-messaging service (SMS) represents a possible intervention to educate concerning PBD benefits and influence dietary behavior change. Indeed, text-messaging interventions have shown promising results in influencing health behavioral change, including increased knowledge and eating behavior among college students, higher adherence to dietary guideline recommendations in patients with coronary heart disease, increased healthy eating behaviors among university students, and decreased red meat consumption among young adults [24-27].

Despite the need to increase PBD and the potential of text messages to influence dietary knowledge and behaviors, few studies have investigated text messages as a strategy to encourage plant-protein consumption. Previous studies have focused on either increasing consumption of fruits and vegetables or decreasing consumption of red meat rather than PBD collectively, and few have explored the effect of environmental sustainability messaging [28]. As environmental impacts of diet are generally less well-known, sustainability messaging may be an effective strategy to influence diet [21,22]. Interestingly, courses focusing on the environmental impact of food have influenced food intake in young adults even more than health-focused courses [29,30]. Furthermore, sustainability messages have 
shown promise in influencing diet quality of young adults [31,32]. Considering the growing interest in environmental sustainability—especially among young adults $[9,10]$-this approach merits further investigation.

\section{Theoretical Framework}

Both the Health Belief Model (HBM) and the Theory of Planned Behavior (TPB) have been effective in promoting health behavior change [33-35]. The HBM is based on the desire to avoid sickness or get well if already ill and the belief that a specific health action will prevent or cure the sickness [36]. Thus, an individual's course of action depends on how they perceive the benefits of adopting the behavior and the consequences of failure to change. It follows that messages targeting these beliefs may influence the desired behavior. The HBM identifies perceived benefits, perceived susceptibility, perceived severity, cues to action (factors that remind individuals to adopt healthy behavior), and self-efficacy as predictors of health behaviors [36]. Constructs from HBM have informed effective change across different behaviors and media, including behaviors associated with diet-related stomach cancer via a text-messaging intervention and osteoporosis prevention via an educational program $[37,38]$. The Theory of Planned Behavior (TPB), previously known as the Theory of Reasoned Action, predicts an individual's intention to engage in a behavior [39]. It states that behavioral achievement is the result of attitudes (which are formed, at least in part, from underlying beliefs about the behavior), perceived behavioral control (similar to self-efficacy in that both measure belief in one's control over the behavior, but operationalized slightly differently [40]), and subjective norms (belief whether the behavior is approved or disapproved by the people of importance to the person) [39]. In addition to the traditional TPB constructs, others have proposed additional factors that uniquely increase the prediction of behavior. For example, several authors have proposed that moral norm or moral satisfaction adds unique predictive power beyond TPB constructs in explaining food intake intentions [41,42]. Of note, text message interventions are generally more influential when multiple behavior-change techniques are incorporated [33].

\section{The Current Study}

Targeting theoretical constructs from HBM and TPB, we designed and compared the effect of two parallel text-message interventions (health-focused and environment-focused) on influencing dietary intention and behavior, with a focus on protein selection. Specifically, the intervention contained messages describing health or environmental benefits of PBD, potential health or environmental consequences of animal-based diets, practical strategies to increase plant-based eating (including links to supportive websites in each message), and information about the increasing popularity of PBD among Americans. Our first objective was to identify the effect of the 8-week text-message intervention (health-focused or environmental focused) on potential dietary predictors (i.e., values, PBD beliefs, subjective norm, self-efficacy, and moral satisfaction), intentions, and actual intake.

In addition to exploring the effectiveness of the intervention itself, we also examined relationships among dietary predictors, intentions, and actual intake. Because environmental, ethical, and health values may influence intention and moderate the effect of other predictors [43-45], we explored potential direct and indirect roles of values on intentions and behaviors. Furthermore, due to the documented role of perceived benefits, perceived susceptibility, perceived severity, subjective norms, and moral satisfaction in explaining dietary and health-related behavior changes [34,35,37,45-48], we explored the extent by which these predictors explained changes in intentions and intake.

\section{Methodology}

This study consisted of four phases: recruiting and screening, baseline data collection (one survey and two dietary assessments), an eight-week text-message intervention, and post-intervention data collection (one survey and two dietary assessments). 


\subsection{Participants}

Young adults between 18 to 26 years old within the United States were recruited via online platforms, including Facebook, Amazon mechanical Turk, and FindParticipants.com. To qualify, participants indicated that they could understand and read English without assistance, owned a mobile phone, were an active user of SMS, were responsible for at least half of their meal choices (purchases, preparation etc.), and consumed both plant-based and animal-based foods. Participants were excluded if they indicated they followed a vegan or vegetarian diet (e.g., lacto-vegetarian, ovo-vegetarian, lacto-ovo-vegetarian, or pescatarian), were planning to change their diet in the next 3 months (e.g., trying to lose weight), or had a history of eating disorder(s). Using standard error estimates for protein intake among males and females ages 20-29 [49], we calculated a sample size of at least 68 participants per group would be required to detect a difference of one serving of protein (approximately $25 \mathrm{~g}$ ) with $80 \%$ power at an alpha of 0.05 .

A sample of 505 participants met the initial screening criteria. All of the interested participants provided informed consent before participating in the study. Of the initial participants, 201 successfully completed the baseline survey and diet assessments. Following completion of baseline surveys, participants were randomly assigned to one of two text message interventions (described below). Of these participants, 159 completed the study (see Figure 1). Participants were awarded an e-gift card both after completing the initial surveys and after completing the final surveys.

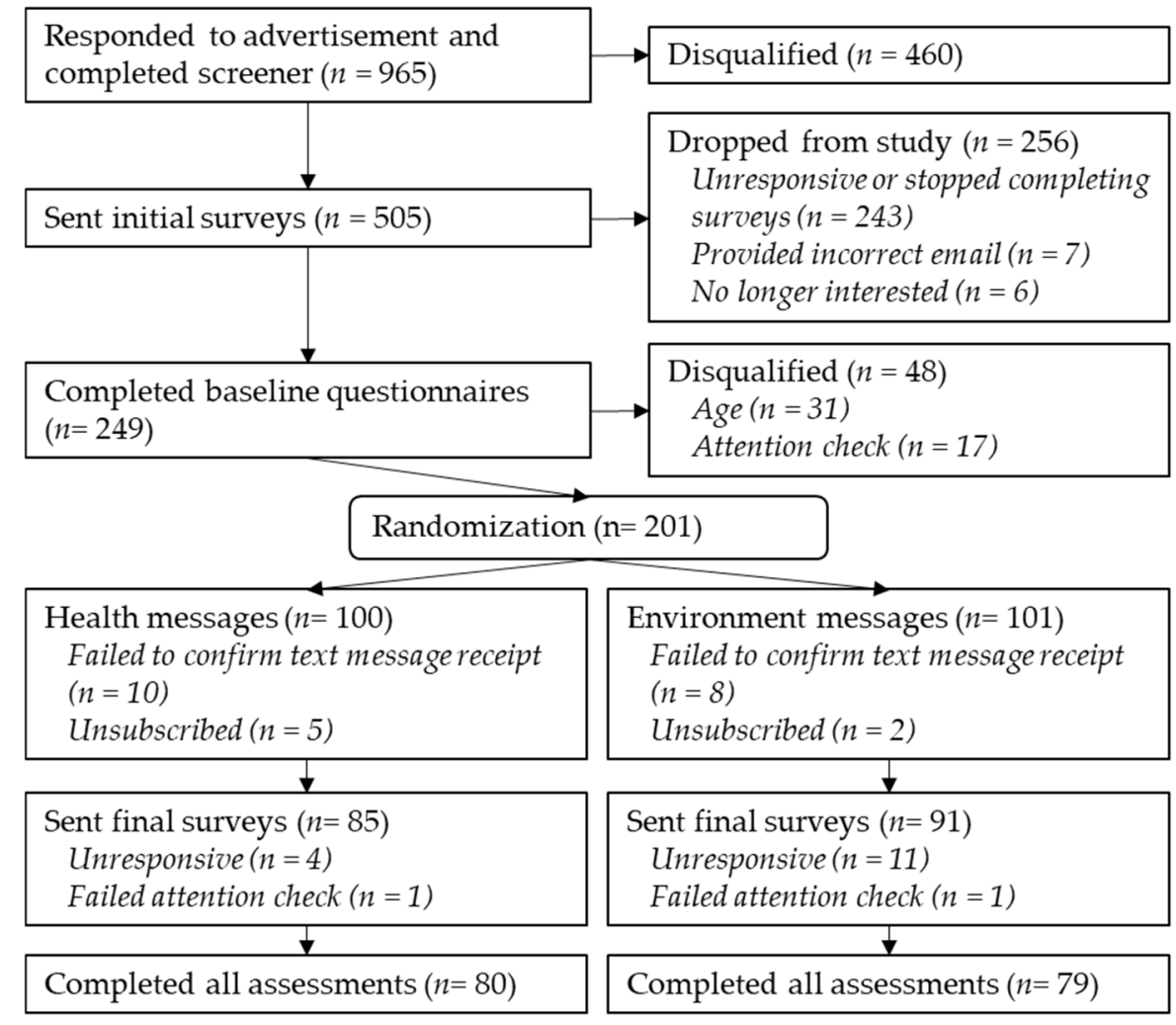

Figure 1. Flowchart of study participants.

\subsection{Survey Instruments}

\subsubsection{Dietary Assessment}

Following study enrollment, participants were emailed an invitation to complete two dietary assessment surveys on two unannounced days (one weekday, one weekend day). Dietary intake data were collected and analyzed using the Automated Self-Administered 24-h (ASA24) Dietary Assessment Tool, version 2020, developed by the National Cancer Institute (Bethesda, MD, USA), which has been validated for use in large-scale nutrition 
research [50,51]. The ASA24 guides participants through a series of questions to assess the foods and beverages that were consumed the previous day. Participants who failed to complete the assessment the day it was received were emailed an unannounced invitation on a later day. Participants also completed two additional ASA24 dietary assessments immediately following the text-message intervention. For the purpose of this study, animalbased foods refer to meat (beef, pork, goat, lamb, and venison), poultry (chicken, turkey, and bird), seafood (fish and shellfish), eggs, dairy, and their manufactured products. Plantbased foods refer to fruits, vegetables (dark-green vegetables, red and orange vegetables, legumes, starchy vegetables, and other vegetables), whole grains, nuts, seeds, and their manufactured products.

\subsubsection{Study Questionnaire}

The survey questionnaire was administered online via survey software. Participants answered questions regarding their personal values (health and green consumer values), health- and environment-focused questions concerning PBD beliefs (perceived benefits of consumption; perceived severity of and susceptibility to consequences of not consuming), self-efficacy, subjective norms, and moral satisfaction using 7-point Likert scales anchored by strongly disagree and strongly agree. Participants also stated their intention to consume plant-based proteins, fruits and vegetables, and animal-based protein sources using a 100-point sliding scale. Survey items were adapted from previously established valid and reliable instruments $[34,35,52,53]$ (Appendix A, Table A1). To assess content validity, we ensured that the various items covered the construct of interest. Internal consistency of survey items was assessed using Cronbach's alpha as a measure of reliability [54]. Cronbach's alpha coefficient can range from 0.0 to 1.0. A Cronbach's alpha close to 1.0 indicates that the item is considered to have a high internal consistency reliability, above 0.8 is considered good, and 0.7 is considered acceptable [55]. Cronbach's alpha for all constructs was above 0.7 , with the vast majority exceeding 0.890 (Appendix A, Table A1).

One attention check question was also included; data from participants who incorrectly answered the attention check question were removed. General participant characteristics, including age, height, weight, gender, ethnicity, educational level, annual household income, biggest meal of the day, and time zone of place of residence, was also collected. Upon completion of the final dietary assessments, participants were emailed the same survey previously described to collect post-intervention data, with the exclusion of demographic questions.

\subsection{Text Message Intervention}

After participants completed baseline data collection, they were randomly divided into two treatment groups: Health Message (HM) and Environment Message (EM). Minor modifications were made so that the groups were comparable in terms of group size, education, income, age, and gender at baseline. Participants then received an initial text message to confirm their participation in the study. Once participants confirmed receipt of the introductory text message, they began to receive the study text messages. Thirty-two messages were developed: 16 health-focused and 16 environment-focused. The behavioral theory constructs served as guide in developing the SMS. The text messages for each group were comparable in content and structure, with only slight variation in the words related to the corresponding intervention. Text messages were screened for understandability and effectiveness and modified accordingly. Due to the small but significant effect of supplementary materials on text-message effectiveness [33], we also included links to websites to aid participants in PBD implementation. The complete list of SMS can be found in Appendix A, Table A2.

Participants received 2-3 SMS per week for eight consecutive weeks on Tuesdays (a weekday) and Fridays (preceding the weekend) via Textedly (SMS marketing software; textedly.com). The participants received the SMS at either 11:30 am or 4:30 pm depending on their largest meal as indicated in the pre-survey, as previous studies indicated that 
SMS are most useful for behavior change when received at high-risk situations [24,56]. The majority of the SMS delivery was unidirectional except for a biweekly SMS asking participants to text back 1 (not at all), 2 (maybe), or 3 (likely) to "How likely are you to focus on eating plant-based protein next week?" and "How likely are you to focus on eating more fruit and vegetables next week?"

\subsection{Statistical Analysis}

Comparability of participant characteristics between groups was assessed using the chi-square statistic. To explore the effects of the text-message interventions on dietary predictors, intentions, and behaviors, we conducted paired-samples $t$-tests using baseline and post-intervention responses. To explore potential differences between those assigned to health-focused vs. environment-focused text messages, an independent samples $t$-test of changes in outcomes (post data-baseline data) between the two groups was conducted. Correlations between changes in outcomes and education and income were calculated using the Pearson's correlation. Gender differences were compared using an independent samples $t$-test of changes in outcomes. Due to a small sample size, the "other" gender category was not included in the analysis.

Considering the exploratory nature of this study, we investigated the effect of the collective text-message intervention on relationships among predictor variables and outcomes using two analyses. First, we examined whether baseline data predicted changes in outcome variables by regressing the post-intervention outcome variable on the baseline predictor variable while controlling for the baseline value of the outcome variable, as suggested by Cole and Maxwell for mediation analysis of half-longitudinal data [57]. Group (health vs. environment) was also included in the model to identify possible differences between HM and EM. As a second analysis, we used the Pearson's correlation coefficient to explore relationships among changes in predictors, intentions, and intake.

In all analyses, no adjustments were made for multiple comparisons. For analyses including predictor variables, only data from the survey instrument that corresponded with the intervention were included (e.g., health values were used as "values" for the $\mathrm{HM}$, and green consumer values were used as "values" for the EM). All analyses were conducted using SPSS version 26.

\section{Results}

The groups were comparable in terms of group size, gender, education, and income, as shown in Table 1.

Due to the exploratory nature of this study, a number of comparisons and analyses were conducted, and thus, results should be interpreted within context of the study's objectives. In the subsequent results and discussion, we use "marginally significant" when $p<0.05$ and "significant" to describe results where $p<0.01$. Greater emphasis is given to significant results and/or consistent trends that were marginally significant across multiple variables.

\subsection{Effect of SMS on Predictor Variables and Dietary Outcomes}

Our first objective was to explore and compare the collective and individual effect of the health-focused and environmental-focused eight-week text-message interventions on PBD beliefs, subjective norms, self-efficacy, moral satisfaction, dietary intentions, and actual intake of protein foods and fruits and vegetables. Because no group differences $(p<0.05)$ were detected when directly comparing changes in variables using an independent samples $t$-test (data not shown), the data were combined for a single analysis, and results for each group are displayed for individual comparison (Table 2). Following the text message intervention, we observed a significant increase in moral satisfaction and perceived benefits of PBD and a marginally significant increase in perceived susceptibility and self-efficacy. Although we initially assumed values to be a stable state [58], we observed a marginally significant increase in values, which appears to be driven largely by a significant increase 
in green consumer values only within the group that received the environment-focused text messages; no differences were observed in health values in either group. Upon further comparison of differences in each group, only perceived susceptibility was marginally higher in the group receiving the health messages.

Table 1. Participant characteristics.

\begin{tabular}{|c|c|c|c|c|}
\hline Demographic & Total & HM & EM & $\begin{array}{l}\text { Pearson } \chi^{2} \\
\text { Sig. }\end{array}$ \\
\hline \multicolumn{5}{|l|}{ Gender } \\
\hline Female & 107 & 55 & 52 & \multirow{3}{*}{0.743} \\
\hline Male & 49 & 23 & 26 & \\
\hline Other & 3 & 2 & 1 & \\
\hline \multicolumn{5}{|l|}{ Education Levels } \\
\hline Less than high school degree & 0 & 0 & 0 & \multirow{7}{*}{0.244} \\
\hline High school graduate (high school diploma or equivalent including GED) & 9 & 4 & 5 & \\
\hline Some college but no degree & 39 & 14 & 25 & \\
\hline Associate degree in college (2-year) & 9 & 6 & 3 & \\
\hline Bachelor's degree in college (4-year) & 50 & 28 & 22 & \\
\hline Some graduate (Master's, doctorate) or professional (MD, JD, etc.) & 30 & 14 & 16 & \\
\hline Graduate or professional degree & 22 & 14 & 8 & \\
\hline \multicolumn{5}{|l|}{ Annual Income } \\
\hline$\$ 0-\$ 24,999$ & 33 & 11 & 22 & \multirow{6}{*}{0.296} \\
\hline$\$ 25,000-\$ 49,999$ & 42 & 22 & 20 & \\
\hline$\$ 50,000-74,999$ & 25 & 13 & 12 & \\
\hline$\$ 75,000-\$ 99,999$ & 19 & 12 & 7 & \\
\hline$\$ 100,000-\$ 149,000$ & 24 & 12 & 12 & \\
\hline$\$ 150,000$ or more & 16 & 10 & 6 & \\
\hline Total & 159 & 80 & 79 & \\
\hline
\end{tabular}

Table 2. Mean values and changes in dietary predictors, intentions, intakes, and intentions following the intervention. Bold values indicate $p<0.05$. Dashed lines indicate $p<0.001$. Food intake is reported as number of servings.

\begin{tabular}{|c|c|c|c|c|c|c|c|c|c|c|c|c|}
\hline & \multicolumn{4}{|c|}{ Total } & \multicolumn{4}{|c|}{ Health Messages (HM) } & \multicolumn{4}{|c|}{ Environment Messages (EM) } \\
\hline & $\begin{array}{c}\mathrm{T} 1 \\
\text { (SD) }\end{array}$ & $\begin{array}{c}\mathrm{T} 2 \\
(\mathrm{SD})\end{array}$ & $\Delta$ & $p$ & $\begin{array}{c}\mathrm{T} 1 \\
\text { (SD) }\end{array}$ & $\begin{array}{c}\mathrm{T} 2 \\
(\mathrm{SD})\end{array}$ & $\Delta$ & $p$ & $\begin{array}{c}\mathrm{T} 1 \\
\text { (SD) }\end{array}$ & $\begin{array}{c}\mathrm{T} 2 \\
(\mathrm{SD})\end{array}$ & $\Delta$ & $p$ \\
\hline Values & $\begin{array}{c}5.32 \\
(1.03)\end{array}$ & $\begin{array}{c}5.44 \\
(0.93)\end{array}$ & 0.11 & 0.048 & $\begin{array}{c}5.72 \\
(0.82)\end{array}$ & $\begin{array}{c}5.70 \\
(0.87)\end{array}$ & -0.02 & 0.743 & $\begin{array}{c}4.92 \\
(1.08)\end{array}$ & $\begin{array}{c}5.17 \\
(0.92)\end{array}$ & 0.25 & 0.007 \\
\hline Health value & $\begin{array}{c}5.69 \\
(0.84)\end{array}$ & $\begin{array}{c}5.69 \\
(0.85)\end{array}$ & 0.00 & 0.924 & $\begin{array}{c}5.72 \\
(1.05)\end{array}$ & $\begin{array}{c}5.70 \\
(0.87)\end{array}$ & -0.02 & 0.743 & $\begin{array}{c}5.66 \\
(0.86)\end{array}$ & $\begin{array}{c}5.69 \\
(0.83)\end{array}$ & 0.03 & 0.654 \\
\hline $\begin{array}{l}\text { Green consumer } \\
\text { value }\end{array}$ & $\begin{array}{c}5.02 \\
(1.07)\end{array}$ & $\begin{array}{c}5.18 \\
(1.04) \\
\end{array}$ & 0.16 & 0.007 & $\begin{array}{c}5.12 \\
(0.82)\end{array}$ & $\begin{array}{c}5.19 \\
(1.16)\end{array}$ & 0.07 & 0.362 & $\begin{array}{c}4.92 \\
(1.08)\end{array}$ & $\begin{array}{c}5.17 \\
(0.92)\end{array}$ & 0.25 & 0.007 \\
\hline $\begin{array}{c}\text { Moral } \\
\text { satisfaction }\end{array}$ & $\begin{array}{c}4.41 \\
(1.52)\end{array}$ & $\begin{array}{c}4.81 \\
(1.40)\end{array}$ & 0.39 & - & $\begin{array}{c}4.35 \\
(1.56)\end{array}$ & $\begin{array}{c}4.74 \\
(1.47)\end{array}$ & 0.39 & 0.009 & $\begin{array}{c}4.47 \\
(1.47)\end{array}$ & $\begin{array}{c}4.87 \\
(1.34)\end{array}$ & 0.40 & 0.002 \\
\hline $\begin{array}{l}\text { Perceived } \\
\text { benefits }\end{array}$ & $\begin{array}{c}5.24 \\
(1.28)\end{array}$ & $\begin{array}{c}5.54 \\
(1.26)\end{array}$ & 0.30 & - & $\begin{array}{c}5.20 \\
(1.34)\end{array}$ & $\begin{array}{c}5.53 \\
(1.36)\end{array}$ & 0.33 & 0.010 & $\begin{array}{c}5.29 \\
(1.23)\end{array}$ & $\begin{array}{c}5.56 \\
(1.15)\end{array}$ & 0.27 & 0.011 \\
\hline $\begin{array}{c}\text { Perceived } \\
\text { susceptibility }\end{array}$ & $\begin{array}{c}4.13 \\
(1.47)\end{array}$ & $\begin{array}{c}4.38 \\
(1.52)\end{array}$ & 0.26 & 0.017 & $\begin{array}{c}4.22 \\
(1.51)\end{array}$ & $\begin{array}{c}4.55 \\
(1.51)\end{array}$ & 0.33 & 0.028 & $\begin{array}{c}4.03 \\
(1.43)\end{array}$ & $\begin{array}{c}4.21 \\
(1.52)\end{array}$ & 0.18 & 0.241 \\
\hline $\begin{array}{l}\text { Perceived } \\
\text { severity }\end{array}$ & $\begin{array}{c}5.36 \\
(1.18)\end{array}$ & $\begin{array}{c}5.49 \\
(1.20)\end{array}$ & 0.12 & 0.111 & $\begin{array}{c}6.00 \\
(0.86)\end{array}$ & $\begin{array}{c}6.08 \\
(0.97)\end{array}$ & 0.08 & 0.387 & $\begin{array}{c}4.73 \\
(1.12)\end{array}$ & $\begin{array}{c}4.89 \\
(1.11)\end{array}$ & 0.16 & 0.180 \\
\hline Subjective norms & $\begin{array}{c}4.34 \\
(1.54)\end{array}$ & $\begin{array}{c}4.51 \\
(1.50)\end{array}$ & 0.17 & 0.089 & $\begin{array}{c}4.43 \\
(1.57)\end{array}$ & $\begin{array}{c}4.67 \\
(1.46)\end{array}$ & 0.25 & 0.103 & $\begin{array}{c}4.25 \\
(1.51)\end{array}$ & $\begin{array}{c}4.34 \\
(1.52)\end{array}$ & 0.09 & 0.490 \\
\hline Self-efficacy & $\begin{array}{c}5.40 \\
(1.15)\end{array}$ & $\begin{array}{c}5.56 \\
(1.11)\end{array}$ & 0.16 & 0.031 & $\begin{array}{c}5.53 \\
(1.15)\end{array}$ & $\begin{array}{c}5.68 \\
(1.10)\end{array}$ & 0.15 & 0.185 & $\begin{array}{c}5.26 \\
(1.15)\end{array}$ & $\begin{array}{c}5.43 \\
(1.12)\end{array}$ & 0.17 & 0.080 \\
\hline
\end{tabular}


Table 2. Cont.

\begin{tabular}{|c|c|c|c|c|c|c|c|c|c|c|c|c|}
\hline \multirow[b]{2}{*}{$\begin{array}{l}\text { Total protein } \\
\text { foods }\end{array}$} & \multicolumn{4}{|c|}{ Total } & \multicolumn{4}{|c|}{ Health Messages (HM) } & \multicolumn{4}{|c|}{ Environment Messages (EM) } \\
\hline & $\begin{array}{c}5.44 \\
(3.40)\end{array}$ & $\begin{array}{c}5.74 \\
(3.98)\end{array}$ & 0.30 & 0.341 & $\begin{array}{c}5.03 \\
(3.09)\end{array}$ & $\begin{array}{c}5.65 \\
(3.77)\end{array}$ & 0.62 & 0.182 & $\begin{array}{c}5.85 \\
(3.66)\end{array}$ & $\begin{array}{c}5.84 \\
(4.21)\end{array}$ & -0.02 & 0.969 \\
\hline $\begin{array}{l}\text { Meat, poultry, } \\
\text { seafood }\end{array}$ & $\begin{array}{c}4.06 \\
(3.07)\end{array}$ & $\begin{array}{c}4.29 \\
(3.54)\end{array}$ & 0.23 & 0.431 & $\begin{array}{c}3.81 \\
(2.67)\end{array}$ & $\begin{array}{c}4.20 \\
(3.40)\end{array}$ & 0.39 & 0.328 & $\begin{array}{c}4.31 \\
(3.42)\end{array}$ & $\begin{array}{c}4.39 \\
(3.71)\end{array}$ & 0.07 & 0.868 \\
\hline Meat & $\begin{array}{c}1.14 \\
(1.85)\end{array}$ & $\begin{array}{c}1.13 \\
(1.78)\end{array}$ & -0.02 & 0.936 & $\begin{array}{c}1.13 \\
(1.72)\end{array}$ & $\begin{array}{c}1.03 \\
(1.49)\end{array}$ & -0.10 & 0.698 & $\begin{array}{l}1.16 \\
(2.00)\end{array}$ & $\begin{array}{c}1.22 \\
(2.04)\end{array}$ & 0.07 & 0.801 \\
\hline Poultry & $\begin{array}{c}1.64 \\
(1.98)\end{array}$ & $\begin{array}{l}1.74 \\
(2.26)\end{array}$ & 0.10 & 0.644 & $\begin{array}{c}1.54 \\
(1.81)\end{array}$ & $\begin{array}{c}1.82 \\
(2.40)\end{array}$ & 0.28 & 0.292 & $\begin{array}{l}1.75 \\
(2.15)\end{array}$ & $\begin{array}{l}1.66 \\
(2.12)\end{array}$ & -0.09 & 0.794 \\
\hline Seafood & $\begin{array}{c}0.98 \\
(1.22)\end{array}$ & $\begin{array}{c}0.72 \\
(1.87)\end{array}$ & -0.25 & 0.146 & $\begin{array}{c}0.89 \\
(1.22)\end{array}$ & $\begin{array}{c}0.58 \\
(1.29)\end{array}$ & -0.30 & 0.118 & $\begin{array}{c}1.07 \\
(1.22)\end{array}$ & $\begin{array}{c}0.87 \\
(2.32)\end{array}$ & -0.20 & 0.486 \\
\hline Eggs & $\begin{array}{c}0.26 \\
(0.64)\end{array}$ & $\begin{array}{c}0.66 \\
(0.95)\end{array}$ & 0.40 & - & $\begin{array}{c}0.21 \\
(0.57)\end{array}$ & $\begin{array}{c}0.62 \\
(0.79)\end{array}$ & 0.41 & - & $\begin{array}{c}0.31 \\
(0.71)\end{array}$ & $\begin{array}{c}0.70 \\
(1.10)\end{array}$ & 0.39 & 0.012 \\
\hline Nuts and seeds & $\begin{array}{c}0.54 \\
(0.94)\end{array}$ & $\begin{array}{c}0.50 \\
(0.90)\end{array}$ & -0.04 & 0.622 & $\begin{array}{c}0.48 \\
(0.70)\end{array}$ & $\begin{array}{c}0.53 \\
(0.96)\end{array}$ & 0.05 & 0.640 & $\begin{array}{c}0.60 \\
(1.13)\end{array}$ & $\begin{array}{c}0.47 \\
(0.85)\end{array}$ & -0.12 & 0.296 \\
\hline Legumes & $\begin{array}{c}0.45 \\
(0.89)\end{array}$ & $\begin{array}{c}0.50 \\
(0.97)\end{array}$ & 0.05 & 0.593 & $\begin{array}{c}0.46 \\
(0.88)\end{array}$ & $\begin{array}{c}0.49 \\
(0.93)\end{array}$ & 0.03 & 0.839 & $\begin{array}{c}0.43 \\
(0.90)\end{array}$ & $\begin{array}{c}0.51 \\
(1.02)\end{array}$ & 0.08 & 0.594 \\
\hline Soy & $\begin{array}{c}0.18 \\
(0.63)\end{array}$ & $\begin{array}{c}0.29 \\
(0.77)\end{array}$ & 0.12 & 0.145 & $\begin{array}{c}0.18 \\
(0.69)\end{array}$ & $\begin{array}{c}0.30 \\
(0.77)\end{array}$ & 0.12 & 0.283 & $\begin{array}{c}0.17 \\
(0.56)\end{array}$ & $\begin{array}{c}0.28 \\
(0.78)\end{array}$ & 0.11 & 0.328 \\
\hline Total dairy & $\begin{array}{c}1.46 \\
(1.41)\end{array}$ & $\begin{array}{c}1.31 \\
(1.24)\end{array}$ & -0.15 & 0.234 & $\begin{array}{l}1.55 \\
(1.69)\end{array}$ & $\begin{array}{c}1.45 \\
(1.42)\end{array}$ & -0.10 & 0.592 & $\begin{array}{l}1.36 \\
(1.06)\end{array}$ & $\begin{array}{c}1.17 \\
(1.01)\end{array}$ & -0.19 & 0.233 \\
\hline Total fruits & $\begin{array}{c}0.80 \\
(0.93)\end{array}$ & $\begin{array}{c}0.82 \\
(0.96)\end{array}$ & 0.02 & 0.801 & $\begin{array}{c}0.79 \\
(0.97)\end{array}$ & $\begin{array}{c}0.82 \\
(1.05)\end{array}$ & 0.03 & 0.793 & $\begin{array}{c}0.80 \\
(0.89)\end{array}$ & $\begin{array}{c}0.81 \\
(0.88)\end{array}$ & 0.01 & 0.934 \\
\hline Total vegetables & $\begin{array}{l}1.43 \\
(0.98)\end{array}$ & $\begin{array}{c}1.61 \\
(1.05)\end{array}$ & 0.17 & 0.068 & $\begin{array}{l}1.50 \\
(1.06)\end{array}$ & $\begin{array}{l}1.50 \\
(0.93)\end{array}$ & 0.00 & 0.999 & $\begin{array}{c}1.37 \\
(0.90)\end{array}$ & $\begin{array}{c}1.72 \\
(1.16)\end{array}$ & 0.35 & 0.015 \\
\hline Total grains & $\begin{array}{c}6.75 \\
(3.03)\end{array}$ & $\begin{array}{c}6.96 \\
(4.57)\end{array}$ & 0.20 & 0.578 & $\begin{array}{c}6.69 \\
(2.74)\end{array}$ & $\begin{array}{c}6.75 \\
(4.23)\end{array}$ & 0.07 & 0.902 & $\begin{array}{c}6.82 \\
(3.32)\end{array}$ & $\begin{array}{c}7.17 \\
(4.92)\end{array}$ & 0.35 & 0.501 \\
\hline Whole grains & $\begin{array}{c}1.16 \\
(1.69)\end{array}$ & $\begin{array}{l}1.54 \\
(2.64)\end{array}$ & 0.38 & 0.069 & $\begin{array}{c}0.96 \\
(0.86)\end{array}$ & $\begin{array}{l}1.55 \\
(3.14)\end{array}$ & 0.58 & 0.099 & $\begin{array}{l}1.36 \\
(2.22)\end{array}$ & $\begin{array}{c}1.54 \\
(2.02)\end{array}$ & 0.18 & 0.430 \\
\hline Plant protein & $\begin{array}{c}1.16 \\
(1.39)\end{array}$ & $\begin{array}{l}1.29 \\
(1.79)\end{array}$ & 0.13 & 0.431 & $\begin{array}{c}1.12 \\
(1.34)\end{array}$ & $\begin{array}{c}1.32 \\
(1.97)\end{array}$ & 0.20 & 0.400 & $\begin{array}{l}1.20 \\
(1.45)\end{array}$ & $\begin{array}{c}1.27 \\
(1.60)\end{array}$ & 0.05 & 0.811 \\
\hline $\begin{array}{l}\text { Plant protein } \\
\text { intention }\end{array}$ & $\begin{array}{l}62.06 \\
(27.92)\end{array}$ & $\begin{array}{l}64.42 \\
(26.49)\end{array}$ & 2.36 & 0.222 & $\begin{array}{l}61.34 \\
(26.56)\end{array}$ & $\begin{array}{c}62.72 \\
(26.75)\end{array}$ & 1.38 & 0.623 & $\begin{array}{l}62.77 \\
(29.37)\end{array}$ & $\begin{array}{c}66.11 \\
(26.28)\end{array}$ & 3.34 & 0.214 \\
\hline $\begin{array}{l}\text { Animal protein } \\
\text { intention }\end{array}$ & $\begin{array}{l}75.22 \\
(26.15)\end{array}$ & $\begin{array}{c}65.17 \\
(29.33)\end{array}$ & -10.05 & - & $\begin{array}{l}75.68 \\
(26.19)\end{array}$ & $\begin{array}{c}63.55 \\
(27.84)\end{array}$ & -12.13 & - & $\begin{array}{l}74.76 \\
(26.28)\end{array}$ & $\begin{array}{c}66.83 \\
(30.87)\end{array}$ & -7.92 & 0.013 \\
\hline $\begin{array}{l}\text { Fruits, veg, } \\
\text { intention }\end{array}$ & $\begin{array}{l}85.30 \\
(18.35)\end{array}$ & $\begin{array}{l}87.50 \\
(15.42)\end{array}$ & 2.21 & 0.050 & $\begin{array}{c}85.51 \\
(18.19)\end{array}$ & $\begin{array}{c}88.33 \\
(14.53)\end{array}$ & 2.81 & 0.098 & $\begin{array}{l}85.08 \\
(18.62)\end{array}$ & $\begin{array}{c}86.67 \\
(16.31)\end{array}$ & 1.60 & 0.283 \\
\hline
\end{tabular}

Consistent with our hypotheses, intentions to consume animal-based protein sources significantly decreased while intentions to consume fruits and vegetables marginally increased following the intervention. Although the study was originally designed with the goal to increase plant-based protein intake, the increase in intentions to consume plant protein foods did not reach statistical significance. Despite intentions to increase fruit and vegetable intake and decrease animal-based protein intake, significant changes in actual food intake servings were only observed for eggs. Interestingly, a marginal increase in vegetable intake was observed only in the group that received the environmentfocused text messages. We also assessed whether changes differed by gender, education, and income. Higher education marginally correlated with increased meat, poultry, and seafood intake. Income was positively correlated with changes in legume intake and marginally negatively correlated with changes in intention to consume animal foods. 
Females marginally consumed more seafood, while males marginally consumed more eggs (Table 3).

Table 3. Correlations between education, income, and changes in study outcomes and gender differences between changes in study outcomes ( $\Delta$ indicates change in females-change in males; thus, positive numbers indicate greater changes in females). Bold values indicate $p<0.05$.

\begin{tabular}{|c|c|c|c|c|c|c|}
\hline & \multicolumn{2}{|c|}{ Education } & \multicolumn{2}{|c|}{ Income } & \multicolumn{2}{|c|}{ Gender } \\
\hline & $r$ & $p$ & $r$ & $p$ & $\Delta$ & $p$ \\
\hline Values & -0.055 & 0.487 & 0.003 & 0.970 & -0.130 & 0.425 \\
\hline Moral satisfaction & 0.109 & 0.171 & 0.153 & 0.053 & -0.266 & 0.199 \\
\hline Perceived benefits & 0.045 & 0.576 & 0.098 & 0.221 & -0.279 & 0.108 \\
\hline Perceived susceptibility & 0.091 & 0.256 & 0.157 & 0.048 & -0.152 & 0.567 \\
\hline Perceived severity & 0.083 & 0.296 & -0.094 & 0.239 & -0.139 & 0.405 \\
\hline Subjective norms & 0.145 & 0.068 & 0.040 & 0.620 & -0.079 & 0.689 \\
\hline Self-efficacy & 0.061 & 0.441 & -0.047 & 0.557 & -0.086 & 0.600 \\
\hline Total protein & 0.183 & 0.021 & -0.042 & 0.599 & -0.318 & 0.687 \\
\hline Meat, poultry, and seafood & 0.202 & 0.011 & -0.006 & 0.942 & -0.460 & 0.477 \\
\hline Meat & 0.128 & 0.109 & 0.033 & 0.677 & -0.170 & 0.715 \\
\hline Poultry & 0.067 & 0.402 & -0.087 & 0.276 & -0.860 & 0.062 \\
\hline Seafood & -0.030 & 0.710 & 0.013 & 0.874 & 0.831 & 0.028 \\
\hline Eggs & 0.151 & 0.058 & 0.020 & 0.807 & -0.497 & 0.047 \\
\hline Soy & -0.020 & 0.801 & -0.056 & 0.481 & 0.174 & 0.436 \\
\hline Nuts and seeds & 0.011 & 0.893 & -0.094 & 0.238 & 0.314 & 0.073 \\
\hline Legumes & -0.059 & 0.462 & 0.224 & 0.005 & -0.455 & 0.083 \\
\hline Total dairy & -0.091 & 0.253 & 0.097 & 0.223 & -0.124 & 0.643 \\
\hline Total fruits & 0.079 & 0.320 & -0.031 & 0.698 & 0.095 & 0.576 \\
\hline Total vegetables & 0.056 & 0.480 & -0.002 & 0.980 & -0.090 & 0.662 \\
\hline Total grains & 0.070 & 0.379 & 0.142 & 0.074 & -1.850 & 0.067 \\
\hline Whole grains & 0.009 & 0.914 & 0.043 & 0.587 & -0.697 & 0.268 \\
\hline Plant protein & -0.044 & 0.584 & 0.061 & 0.446 & 0.025 & 0.954 \\
\hline Plant protein intention & -0.155 & 0.052 & -0.093 & 0.243 & -0.226 & 0.957 \\
\hline Animal food intention & -0.056 & 0.488 & -0.161 & 0.043 & 7.563 & 0.124 \\
\hline Fruits and vegetables intention & 0.008 & 0.918 & 0.021 & 0.795 & -0.942 & 0.700 \\
\hline
\end{tabular}

\subsection{Relationships among Predictor and Outcome Variables}

\subsubsection{Relationships among Baseline Values and Other Predictor Variables}

Due to the direct effect of personal values on food behaviors and mediators of dietary intention [42,45], we next explored the extent by which values influenced dietary predictors, intentions, and behaviors (Table 4). Higher baseline values consistently predicted significantly larger SMS-induced increases in perceived susceptibility, perceived severity, and moral satisfaction and marginal increases in subjective norms and self-efficacy. Interestingly, the largest effect size was observed for perceived severity of failure to consume PBD, which was more pronounced in the group receiving health-focused messages. While baseline values did not predict a change in perceived benefits, changes in values were positively correlated with changes in perceived benefits. Changes in subjective norms also positively marginally correlated with changes in values. 
Table 4. Two independent analyses were conducted to explore relationships among predictor and outcome variables. First, post-intervention variables were individually regressed on baseline predictor variables and group (health vs. environmental text messages). Standardized beta values are displayed. Second, correlations between changes in the predictor variables and changes outcome variables were explored using a Pearson correlation. Bold values indicate $p<0.05$. Dashed lines indicate $p<0.001$.

\begin{tabular}{|c|c|c|c|c|c|c|c|}
\hline Predictor & Outcome & B & $p$ & B (Group) & $p$ (Group) & $r$ & $p$ (Corr.) \\
\hline \multirow{14}{*}{ Values } & $\begin{array}{l}\text { Meat, poultry, and } \\
\text { seafood }\end{array}$ & 0.210 & 0.009 & 0.076 & 0.341 & -0.011 & 0.891 \\
\hline & Meat & -0.018 & 0.831 & 0.046 & 0.590 & 0.119 & 0.134 \\
\hline & Fruits & 0.100 & 0.194 & 0.030 & 0.692 & 0.030 & 0.704 \\
\hline & Vegetables & 0.097 & 0.233 & 0.164 & 0.045 & 0.069 & 0.385 \\
\hline & Plant protein & 0.071 & 0.409 & 0.006 & 0.940 & 0.090 & 0.258 \\
\hline & Plant protein intention & 0.076 & 0.305 & 0.079 & 0.262 & 0.128 & 0.109 \\
\hline & Animal food intention & -0.169 & 0.027 & -0.002 & 0.978 & -0.072 & 0.372 \\
\hline & $\begin{array}{c}\text { Fruits and vegetables } \\
\text { intention }\end{array}$ & 0.069 & 0.291 & -0.019 & 0.766 & 0.033 & 0.683 \\
\hline & Perceived benefits & 0.063 & 0.341 & 0.014 & 0.834 & 0.176 & 0.026 \\
\hline & Perceived susceptibility & 0.185 & 0.010 & -0.005 & 0.936 & 0.026 & 0.749 \\
\hline & Perceived severity & 0.255 & 一 & -0.162 & 0.015 & 0.103 & 0.196 \\
\hline & Subjective norms & 0.148 & 0.036 & -0.020 & 0.754 & 0.166 & 0.037 \\
\hline & Self-efficacy & 0.175 & 0.018 & 0.022 & 0.740 & 0.139 & 0.081 \\
\hline & Moral satisfaction & 0.181 & 0.007 & 0.093 & 0.149 & 0.084 & 0.291 \\
\hline \multirow{8}{*}{ Perceived benefits } & $\begin{array}{l}\text { Meat, poultry, and } \\
\text { seafood }\end{array}$ & 0.019 & 0.804 & -0.006 & 0.941 & -0.007 & 0.935 \\
\hline & Meat & -0.056 & 0.483 & 0.055 & 0.484 & -0.053 & 0.509 \\
\hline & Fruits & 0.099 & 0.163 & -0.012 & 0.868 & -0.090 & 0.257 \\
\hline & Vegetables & 0.040 & 0.604 & 0.125 & 0.098 & -0.026 & 0.744 \\
\hline & Plant protein & 0.155 & 0.048 & -0.027 & 0.728 & 0.015 & 0.856 \\
\hline & Plant protein intention & 0.091 & 0.158 & 0.046 & 0.469 & 0.179 & 0.024 \\
\hline & Animal food intention & -0.049 & 0.489 & 0.066 & 0.345 & -0.082 & 0.305 \\
\hline & $\begin{array}{l}\text { Fruits and vegetables } \\
\text { intention }\end{array}$ & -0.084 & 0.161 & -0.043 & 0.473 & 0.064 & 0.423 \\
\hline \multirow{8}{*}{$\begin{array}{c}\text { Perceived } \\
\text { susceptibility }\end{array}$} & $\begin{array}{l}\text { Meat, poultry, and } \\
\text { seafood }\end{array}$ & 0.156 & 0.037 & 0.004 & 0.959 & -0.105 & 0.188 \\
\hline & Meat & 0.111 & 0.162 & 0.060 & 0.446 & -0.118 & 0.138 \\
\hline & Fruits & -0.009 & 0.902 & -0.009 & 0.902 & -0.004 & 0.961 \\
\hline & Vegetables & -0.091 & 0.233 & 0.123 & 0.105 & 0.124 & 0.119 \\
\hline & Plant protein & 0.022 & 0.779 & -0.020 & 0.796 & 0.090 & 0.262 \\
\hline & Plant protein intention & 0.140 & 0.030 & 0.059 & 0.356 & 0.007 & 0.931 \\
\hline & Animal food intention & -0.070 & 0.330 & 0.059 & 0.396 & -0.107 & 0.183 \\
\hline & $\begin{array}{l}\text { Fruits and vegetables } \\
\text { intention }\end{array}$ & -0.057 & 0.342 & -0.049 & 0.410 & 0.027 & 0.740 \\
\hline
\end{tabular}


Table 4. Cont.

\begin{tabular}{|c|c|c|c|c|c|c|c|}
\hline Predictor & Outcome & B & $p$ & B (Group) & $p$ (Group) & $r$ & $p$ (Corr.) \\
\hline \multirow{8}{*}{$\begin{array}{l}\text { Perceived } \\
\text { severity }\end{array}$} & Meat, poultry, seafood & 0.093 & 0.292 & 0.045 & 0.610 & -0.050 & 0.533 \\
\hline & Meat & 0.029 & 0.759 & 0.068 & 0.464 & -0.057 & 0.477 \\
\hline & Fruits & 0.059 & 0.485 & 0.023 & 0.781 & 0.033 & 0.676 \\
\hline & Vegetables & -0.004 & 0.963 & 0.125 & 0.163 & 0.100 & 0.211 \\
\hline & Plant protein & 0.051 & 0.585 & 0.006 & 0.952 & 0.103 & 0.196 \\
\hline & Plant protein intention & 0.185 & 0.015 & 0.149 & 0.048 & -0.069 & 0.390 \\
\hline & Animal food intention & 0.034 & 0.687 & 0.083 & 0.319 & -0.164 & 0.040 \\
\hline & $\begin{array}{l}\text { Fruits and vegetables } \\
\text { intention }\end{array}$ & 0.041 & 0.562 & -0.024 & 0.740 & -0.089 & 0.263 \\
\hline \multirow{8}{*}{ Subjective norms } & $\begin{array}{l}\text { Meat, poultry, and } \\
\text { seafood }\end{array}$ & 0.164 & 0.028 & 0.003 & 0.967 & -0.049 & 0.541 \\
\hline & Meat & 0.119 & 0.129 & 0.060 & 0.445 & -0.061 & 0.443 \\
\hline & Fruits & 0.027 & 0.700 & -0.007 & 0.925 & -0.049 & 0.541 \\
\hline & Vegetables & 0.100 & 0.185 & 0.133 & 0.078 & 0.008 & 0.925 \\
\hline & Plant protein & 0.069 & 0.386 & -0.017 & 0.827 & 0.082 & 0.302 \\
\hline & Plant protein intention & 0.189 & 0.004 & 0.062 & 0.325 & 0.009 & 0.909 \\
\hline & Animal food intention & -0.150 & 0.038 & 0.054 & 0.438 & -0.062 & 0.438 \\
\hline & $\begin{array}{l}\text { Fruits and vegetables } \\
\text { intention }\end{array}$ & -0.068 & 0.259 & -0.050 & 0.405 & 0.001 & 0.989 \\
\hline \multirow{8}{*}{ Self-efficacy } & Meat, poultry, seafood & 0.103 & 0.170 & 0.007 & 0.928 & -0.043 & 0.593 \\
\hline & Meat & -0.003 & 0.970 & 0.053 & 0.507 & -0.171 & 0.031 \\
\hline & Fruits & 0.099 & 0.164 & 0.003 & 0.961 & -0.056 & 0.482 \\
\hline & Vegetables & 0.053 & 0.493 & 0.133 & 0.081 & 0.029 & 0.718 \\
\hline & Plant protein & 0.165 & 0.036 & -0.001 & 0.985 & $<0.001$ & 0.997 \\
\hline & Plant protein intention & 0.214 & 0.001 & 0.077 & 0.220 & 0.118 & 0.139 \\
\hline & Animal food intention & -0.041 & 0.572 & 0.060 & 0.399 & -0.241 & 0.002 \\
\hline & $\begin{array}{l}\text { Fruits and vegetables } \\
\text { intention }\end{array}$ & 0.002 & 0.974 & -0.046 & 0.450 & 0.092 & 0.250 \\
\hline \multirow{8}{*}{$\begin{array}{c}\text { Moral } \\
\text { satisfaction }\end{array}$} & $\begin{array}{l}\text { Meat, poultry, and } \\
\text { seafood }\end{array}$ & -0.051 & 0.500 & -0.002 & 0.981 & 0.001 & 0.993 \\
\hline & Meat & -0.184 & 0.021 & 0.061 & 0.432 & -0.118 & 0.140 \\
\hline & Fruits & 0.077 & 0.279 & -0.011 & 0.872 & -0.037 & 0.640 \\
\hline & Vegetables & 0.028 & 0.714 & 0.126 & 0.096 & -0.029 & 0.714 \\
\hline & Plant protein & 0.123 & 0.117 & -0.026 & 0.739 & -0.069 & 0.388 \\
\hline & Plant protein intention & 0.147 & 0.023 & 0.045 & 0.479 & 0.083 & 0.298 \\
\hline & Animal food intention & -0.182 & 0.010 & 0.070 & 0.309 & 0.019 & 0.817 \\
\hline & $\begin{array}{l}\text { Fruits and vegetables } \\
\text { intention }\end{array}$ & 0.005 & 0.933 & -0.046 & 0.443 & -0.049 & 0.539 \\
\hline
\end{tabular}

\subsubsection{Relationships among Dietary Intentions and Predictor Variables}

We consistently observed the anticipated relationship between predictor variables and intentions to consume animal (consistently negative) and plant protein (consistently positive) sources in nearly all measured variables (Table 4). Increases in intentions to consume plant-based protein were significantly predicted by subjective norm and self- 
efficacy; marginally predicted by baseline susceptibility, severity, and moral satisfaction; and marginally correlated with increases in perceived benefits. Decreases in intention to consume animal protein sources were predicted by higher baseline subjective norm, moral satisfaction, and values and correlated with increases in perceived severity and self-efficacy. Of note, no variables predicted changes in intention to consume fruits and vegetables.

\subsubsection{Relationships among Actual Intake and Predictor Variables}

Higher baseline perceived benefits of PBD and self-efficacy marginally predicted an increased effect of the SMS intervention on plant protein intake (Table 4). Contrary to our hypothesis, higher baseline perceived susceptibility, subjective norm, and values predicted an increase in the combined category of meat, poultry, and seafood intake. Contrastingly, when meat was analyzed separately, opposite trends were observed: higher moral satisfaction marginally predicted lower meat intake, and meat intake marginally decreased as self-efficacy increased. Although we did not observe strong predictive effects of explanatory variables on fruit or vegetable intake, we did observe a small group effect for values, suggesting that environmental-focused text messages have a greater influence on vegetable intake for those with higher environmental values than do health-focused messages on those with higher health values, consistent with our observation of a greater overall vegetable intake in the environment group (Table 2).

\section{Discussion}

In this study, we used a TPB- and HBM-informed approach to explore the effect of a health-focused and environment-focused text-message intervention on dietary intake and intention, with a focus on protein source. We also explored the possible predictive role of values, PBD beliefs (i.e., perceived PBD benefits, perceived severity of and susceptibility to consequences of not consuming PBD), subjective norms, self-efficacy, and moral satisfaction.

\subsection{Implications for Plant Protein}

Although intentions and actual intake of plant-protein sources did not change as a result of the intervention, all measured predictors significantly or marginally explained increases in plant protein intention and/or intake. Our observation of relatively stronger effect sizes for subjective norms and self-efficacy extends previous findings: while others identified a lack of knowledge and awareness of others' expectations as potential PBD barriers [22], we identified self-efficacy and subjective norms as a potential target to improve intentions. When combined with other strategies, messaging that increases awareness of and practical strategies to incorporate plant-based protein sources may influence PBD adoption.

As acceptance of alternative proteins is affected by food neophobia, the fear of trying new or unfamiliar foods, lack of familiarity with plant-based protein sources may be one explanation for our failure to detect an increase in plant-protein intake [59,60]. Indeed, food neophobia is negatively associated with liking beans and legumes [60]. The unfamiliarity of PBD and plant proteins may have induced reluctance to incorporate plant protein foods and adoption of PBD. Perceived taste is also a barrier to PBD adoption [22], and thus, future studies should explore the impact of messaging on taste perception. Because taste and familiarity are indispensable determinants of acceptance for healthy and sustainable foods [61], interventions that target these attributes may be necessary to increase intake for some populations.

\subsection{Implications for Animal Protein}

The text-message intervention resulted in a meaningful and significant decrease in intentions to consume animal foods. This decrease was explained in part by baseline moral satisfaction, subjective norms, and values and correlated with increases in self-efficacy. Consistent with our findings in plant-based protein intention, our observation that actual 
meat intake decreased as self-efficacy increased suggests that messages targeting abilities to prepare and consume PBD may be an effective strategy to influence protein selection. The effectiveness of messages promoting the normalcy of reducing meat consumption is supported by our data and others' findings that normative meat-eating statements lowered self-reported interest in meat-eating and actual orders of a meatless lunch [46]. Furthermore, our data suggest potential strategies for targeting meat intake among individuals with certain values or beliefs: PBD messages with an ethical focus may resonate with the increasing number of people that consider animal welfare a moral issue [62], while health messages may be more effective for those that perceive the health severity of animal-based diets, consistent with others' observations of greater meat discouragement among those that believed meat was bad for health but not among those that believed it was bad for the environment [17].

Our observation that meat intake but not poultry and seafood intake changed in the expected direction may have several possible explanations. Firstly, these observations support the efficacy of the text-message intervention, as meat was defined for participants as distinct from poultry or seafood, and several text messages mentioned meat specifically, but none mentioned poultry or seafood. Others' finding that over one-third of meatreducers report increasing poultry consumption may also explain our observation [18]. We hypothesize that our observed increase in egg consumption may have a similar explanation: participants may have increased non-meat protein sources in response to the text messages.

\subsection{Health vs. Environmental Messaging}

Although we observed few differences between the EM and HM groups, several observations suggest a small but unique role of environment-focused messages. Firstly, we observed that green consumer values significantly increased only in the EM group and predicted increased vegetable intake to a greater extent than health values in the HM group. Importantly, higher vegetable intake was observed only in the EM group. Furthermore, baseline values consistently explained changes in meaningful predictors of dietary intention and behavior. Low knowledge and awareness of PBD [21], low involvement in sustainable eating compared with healthy eating [63], and lack of evidence regarding poor nutrition knowledge as a predictive factor in healthy eating $[64,65]$ together suggest that environmental (but not health) education may present an opportunity: increasing knowledge about environmental effects of a PBD may alter green values, which in turn may influence dietary choices.

However, we note that no direct group difference was observed for any of the measured outcomes, perhaps due to relatively lower statistical power. Thus, the content of the text message (i.e., health or environment) may be less influential than other factors, such as targeting certain beliefs, attitudes, or self-efficacy. While environment-focused messaging alone would likely be insufficient to influence PBD adoption, we suggest that sustainability messaging may be part an overall strategy to influence plant-based eating among young adults. Furthermore, considering that those involved in sustainable eating tend to also be highly involved in healthy eating (but not necessarily the opposite) and the considerable overlap between perception of healthy, sustainable, and plant-based diets, sustainability messaging would likely be compatible with existing guidelines for healthy eating [63]. More research is needed to understand the unique effect of health vs. environmental messages.

\subsection{Limitations, Strengths and Future Directions}

Despite the convenience, non-disruptive nature, and low cost of text-message interventions, further research is needed to understand how factors, such as frequency, length of intervention, timing of delivery, tone, and duration of the effect, can be optimized $[66,67]$. Furthermore, knowing whether participants actually read and understand the messages is a challenge, similar to other education interventions, such as emails or classes [24]. Evidence from systematic reviews suggest that although text messages can be effective for 
promoting health behaviors, further research is needed to optimize implementation [66]. Potential strategies to optimize text-message interventions include greater interactivity and engagement strategies, such as accessibility to an advisor and text message tone $[33,68]$.

Interpretation of our results should be considered in context of several limitations. Although efforts were taken to balance gender representation in the study sample, greater attrition among males resulted in a disproportionate number of female representation in our sample. We also recognize that most of the effect sizes we observed were relatively small and that long-term persistence of our observations require further study. Although we incorporated a number of relevant behavioral constructs to explain dietary intentions and behavior, the presence of other possible explanatory factors should also be considered, such as self-regulation [28] and self-identity [42]. Importantly, the exploratory nature of the data analysis must also be considered when interpreting our findings. Additional a priori investigations and full mediation analysis are necessary to confirm the results of the current study.

Despite its limitations, this study also provides several novel contributions. While previous studies have focused primarily on decreasing animal-protein intake and increasing fruit and vegetable intake [28], our focus on plant protein adds needed insights, especially considering the increasing popularity of plant-based protein [69]. Of note, we collected dietary intake using a comprehensive and validated tool, thus strengthening our conclusions about the impact of the intervention on actual intake. Importantly, vegans and vegetarians were excluded from the study, and thus, our findings are better applied to populations currently consuming animal-based protein sources.

\section{Conclusions}

In this study, we reveal a possible role of text-messages in decreasing intention to consume animal protein sources and increasing intention to consume fruits and vegetables. However, using a validated dietary assessment tool, we detected significant dietary changes only for egg intake. We also identified several conditions when messaging interventions may be more effective in influencing protein-source intake: individuals with relatively higher self-efficacy regarding their abilities to consume PBD, a feeling of the moral rightness of PBD, and a sense of the subjective normalcy of PBD consumption may be more influenced by messaging campaigns to encourage PBD adoption. While we did not observe a consistent advantage of an environment vs. health-focused messaging campaign, our findings of altered green consumer values and vegetable intake in the EM group suggest that environment-focused messages may be compatible with existing strategies to encourage healthier and more environmentally friendly dietary patterns among young adults.

Author Contributions: Conceptualization, T.J.L. and J.C.K.; methodology, T.J.L. and J.C.K.; formal analysis, J.C.K. and R.N.O.; investigation, T.J.L. and J.C.K.; writing—original draft preparation, T.J.L. and J.C.K.; writing-review and editing, T.J.L., J.C.K. and R.N.O.; visualization, T.J.L., J.C.K. and R.N.O.; supervision, J.C.K.; project administration, T.J.L. and J.C.K. All authors have read and agreed to the published version of the manuscript.

Funding: This research received no external funding.

Institutional Review Board Statement: All procedures were approved by the Bowling Green State University Institutional Review Board, protocol 1694528.

Informed Consent Statement: Informed consent was obtained from all subjects involved in the study.

Data Availability Statement: Data can be found at the following location: doi:10.17632/fwdssw4k3p.1. Additional data are available upon reasonable request.

Acknowledgments: The authors wish to thank Kerri Knippen and Torie Wagner-Greene for their advice in choosing and utilizing appropriate behavior-change theories as well as Wan Shen for providing feedback. We also thank James McGraw for his advice on data analysis. Last but not least, the authors also appreciate Carly Sedlacek for her assistance in managing data collection. 
Conflicts of Interest: The authors declare no conflict of interest.

\section{Appendix A}

Table A1. Example items for food consumption intention, green consumer values, health values, and behavior theory questionnaires. Unless otherwise noted, all questions were measured on a seven-point scale from $1=$ strongly disagree to $7=$ strongly agree.

\begin{tabular}{ccc}
\hline Constructs & Example Item & Cronbach's $\alpha$ \\
\hline & Q1 On a scale of $0 \%$ to $100 \%$, how likely is it that you will regularly \\
eat plant-based protein sources over the next month? & Q $\begin{array}{c}\text { Food consumption intention } \\
\text { (Adapted from [52]) }\end{array}$ & Q2 On a scale of $0 \%$ to $100 \%$, how likely is it that you will regularly \\
& Q3 On a scale of $0 \%$ to $100 \%$, how likely is it that you will regularly \\
eat fruits and vegetables over the next month?
\end{tabular}

Q1 It is important to me that the products I use do not harm the environment.

Q2 I consider the potential environmental impact of my actions when making many of my decisions.

Green consumer values (Adapted Q3 My purchase habits are affected by my concern for our from [53]) environment.

Q4 I am concerned about wasting the resources of our planet.

Q5 I would describe myself as environmentally responsible.

Q6 I am willing to be inconvenienced in order to take actions that are more environmentally friendly.

Q1 Having good health means a lot to me.

Q2 I often think about my health.

Health values (Adapted from [53])

Q3 I think of myself as a person who is interested in healthful foods.

Q4 I consider the potential health impact of my actions when making many of my decisions.

Q5 My purchase habits are affected by my concern for my health.

Q6 I would describe myself as health conscious.

Q7 I am willing to be inconvenienced in order to take actions that are better for my health.

Q1 Shifting my current diet to more plant-based foods is good for my overall health.

Perceived benefits: Health (Adapted from [34,35])
Q2 Eating less animal-based foods will help reduce my chances of heart disease or cancer.

Q3 I have a lot to gain by eating more plant-based foods.

Q4 Eating less animal products is better for my health.

Q1 I can help improve our environment when I shift my current diet to more plant-based foods.

Perceived benefits: Environment Q2 Eating plenty of plant-based foods will protect the environment. (Adapted from $[34,35]$ )

Q3 Eating more plant-based foods is better for the environment.

Q4 If I consume less animal-based products, it will be better for our environment.

Q1 I feel I have a good chance of getting diseases such as heart disease and cancer in the future if I continue eating a lot of animal-based foods.

Perceived susceptibility: Health (Adapted from [34,35])

Q2 My chances of getting diseases such as heart disease and cancer are large if I fail to eat more plant-based foods.

Q3 My physical health will be jeopardized if I fail to eat more plant-based foods.

Q4 If I don't eat more plant-based foods, I think my chances of getting diseases such as heart disease and cancer sometime in the future would be high. 
Table A1. Cont.

\begin{tabular}{|c|c|c|}
\hline Constructs & Example Item & Cronbach's $\alpha$ \\
\hline $\begin{array}{l}\text { Perceived susceptibility: } \\
\text { Environment } \\
\text { (Adapted from }[34,35] \text { ) }\end{array}$ & $\begin{array}{l}\text { Q1 The well-being of the environment will be jeopardized if I fail to } \\
\text { eat more plant-based foods. } \\
\text { Q2 There is a good possibility that I will cause more damage to our } \\
\text { environment if I fail to shift my current diet to more plant-based } \\
\text { foods. } \\
\text { Q3 I worry a lot about our environment if I continue to eat plenty of } \\
\text { animal-based foods. } \\
\text { Q4 Sometime in the future, our environment will be more damaged } \\
\text { if I fail to shift my current diet to more plant-based foods. }\end{array}$ & 0.929 \\
\hline $\begin{array}{l}\text { Perceived severity: Health } \\
\text { (Adapted from }[34,35] \text { ) }\end{array}$ & $\begin{array}{l}\text { Q1 The thought of having diseases such as heart disease and cancer } \\
\text { scares me. } \\
\text { Q2 Having a poor diet would have major consequences on my life. } \\
\text { Q3 Eating a poor diet will negatively impact my life goals. }\end{array}$ & 0.710 \\
\hline $\begin{array}{l}\text { Perceived severity: Environment } \\
\text { (Adapted from }[34,35] \text { ) }\end{array}$ & $\begin{array}{l}\text { Q1 Harming the environment would be very upsetting for me. } \\
\text { Q2 It would be very serious if I my diet harms the environment. } \\
\text { Q3 Our environment would be put in danger if I don't change my } \\
\text { diet. }\end{array}$ & 0.733 \\
\hline
\end{tabular}

Q1 I feel confident in my ability to shift to more plant-based foods, even if it means finding the time to look up recipes.

Q2 I can eat less animal products even if it is an adjustment for me.

Self-Efficacy

(Adapted from [34,35])

Subjective norms: Health

(Adapted from [34,35])

Subjective norms: Environment (Adapted from [34,35])

Q3 I am confident that I can incorporate more plant-based foods in my diet.

Q4 I believe I have the ability to change my current diet for my health.

Q5 I believe I have the ability to change my current diet for the environment.

Q1 Most people who are important to me would approve of me eating more plant-based foods to improve my health.

Q2 Most people who are important to me would want me to eat more plant-based foods to improve my health.

0.896

Q3 Most people who are important to me think I should eat more plant-based foods to improve my health.

Q1 Most people who are important to me would approve of me eating more plant-based foods to protect the environment.

Q2 Most people who are important to me think I should eat more plant-based foods to protect the environment.

Q3 Most people who are important to me would want me to eat more plant-based foods to protect the environment.

Table A2. A Complete List of Text Messages (SMS).

\begin{tabular}{|c|c|c|}
\hline Week & Health Messages $[1,2,4,11,12,70-72]$ & Environment Messages $[1,2,4,14,70,73,74]$ \\
\hline 1 & $\begin{array}{c}\text { Did you know by eating more plant-based products } \\
\text { (like nuts and black beans) and less animal-based } \\
\text { proteins (like beef), you can reduce your risk of getting } \\
\text { heart disease, type } 2 \text { diabetes, hypertension, obesity, } \\
\text { some cancers, and most chronic diseases? } \\
\text { What's a plant-based diet? } \\
\text { Plant-Based Diet (psu.edu, accessed on } 1 \text { March 2021) } \\
\text { Psst ... . Plant proteins cost far less than animal } \\
\text { proteins. It's a twofer that you're protecting your } \\
\text { health and your wallet! } \\
\text { Is plant-based budget friendly? } \\
2 \text { easy, affordable, plant-centered dinners-Harvard } \\
\text { Health }\end{array}$ & $\begin{array}{c}\text { Did you know by eating more plant-based products } \\
\text { (like nuts and black beans) and less animal-based } \\
\text { proteins (like beef), you can reduce your } \\
\text { environmental footprint on the use of land, water, and } \\
\text { fossil energy? } \\
\text { What's a plant-based diet? } \\
\text { Plant-Based Diet (psu.edu, accessed on } 1 \text { March 2021) } \\
\text { Psst ... . Plant proteins cost far less than animal } \\
\text { proteins. It's a twofer that you're protecting your } \\
\text { planet and your wallet! } \\
\text { Is plant-based budget friendly? } \\
2 \text { easy, affordable, plant-centered dinners-Harvard } \\
\text { Health }\end{array}$ \\
\hline
\end{tabular}


Table A2. Cont.

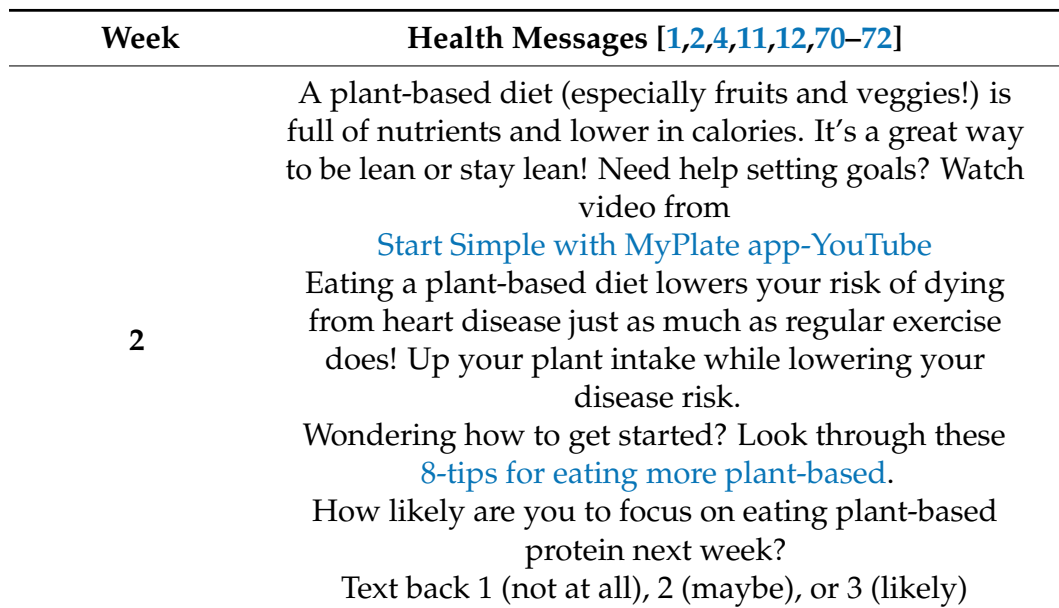

FYI, plants are rich in antioxidants, which protect you from cancer, heart disease, osteoporosis, and enhance your immunity!

Need help taking the first step? Tips>

Enjoy Vegetarian Meals I MyPlate

Health-friendly AND tasty? Try a plant-based burger or other meatless dish and see for yourself! Who knew being nutritious could taste so good?

See these 5 tips for shifting to more plant-based food choices

Environment Messages [1,2,4,14,70,73,74]

A plant-based diet (especially fruits and veggies!)

significantly lowers water and air pollution. It's a great way to be green or stay green!

Need help setting goals? Watch video from

Start Simple with MyPlate app-YouTube

Every serving of plant protein you choose in place of meat saves the pollution of a 100-mile car trip! Up

your plant intake while lowering your environmental foot print.

Wondering how to get started? Look through these 8-tips for eating more plant-based.

How likely are you to focus on eating plant-based protein next week?

Text back 1 (not at all), 2 (maybe), or 3 (likely)

FYI, plant-based foods use far fewer natural resources for production than animal-based foods, which protect our planet from environmental damage! Need help taking the first step? Tips>

Enjoy Vegetarian Meals I MyPlate

Eco-friendly AND tasty? Try a plant-based burger or other meatless dish and see for yourself! Who knew saving the planet could taste so good?

See these 5 tips for shifting to more plant-based food choices

Eating more plant foods is for everyone, not just vegetarians. Improve your health by joining the thousands of people that are eating a plant-forward diet. If you're a meat lover, enjoy it on occasion and in smaller amounts as a garnish. Looking for more plant-based recipes? Explore these resources from the Recipes | MyPlate

Lower your health risk by passing on the extra meat next time. Try veggie days or smaller meat portions.

See these 5 tips for shifting to more plant-based food choices

How likely are you to focus on eating more fruits and vegetables next week?

Text back 1 (not at all), 2 (maybe), or 3 (likely)

Eating too much of animal products and too little of plant products can have major consequences on your health and your life goals. Once you lose your health, you lose what you love to do!

Need help setting goals? Watch video from Start Simple with MyPlate app-YouTube

Animal products have a higher amount of saturated fats. High intakes of saturated fat may lead to the development of cardiovascular disease! Wondering how to get started? Look through these 8-tips for eating more plant-based.
Eating more plant foods is for everyone, not just vegetarians. Protect the environment by joining the thousands of people that are eating a plant-forward diet. If you're a meat lover, enjoy it on occasion and in smaller amounts as a garnish.

Looking for more plant-based recipes? Explore these resources from the Recipes I MyPlate

Lower your environmental impact by passing on the extra meat next time. Try veggie days or smaller meat portions.

See these 5 tips for shifting to more plant-based food choices

How likely are you to focus on eating more fruits and vegetables next week?

Text back 1 (not at all), 2 (maybe), or 3 (likely)

Eating too much of animal products and too little of plant products can have major consequences on our environment and its biodiversity. Once earth loses its biodiversity, we can't get it back!

Need help setting goals? Watch video from Start Simple with MyPlate app-YouTube

Animal agriculture produces higher amounts of harmful nutrient runoff into our lakes and rivers than production of plant-based foods. This environmental pollution destroys wildlife and leads to toxic algae growth! Wondering how to get started? Look through these 8-tips for eating more plant-based. 
Table A2. Cont.

\begin{tabular}{|c|c|c|}
\hline Week & Health Messages $[1,2,4,11,12,70-72]$ & Environment Messages $[1,2,4,14,70,73,74]$ \\
\hline 6 & $\begin{array}{c}\text { Some compounds in meat are converted to a harmful } \\
\text { byproduct, trimethylamine N-oxide (TMAO), by gut } \\
\text { bacteria. High levels of TMAO byproduct are } \\
\text { associated with atherosclerosis, heart attack, and } \\
\text { stroke! Need help taking the first step? Tips > } \\
\text { Enjoy Vegetarian Meals I MyPlate } \\
1 \text { in } 4 \text { Americans ate more plant-based protein last } \\
\text { year. You could do the same! Let's join in. What're you } \\
\text { waiting for? It's about time to make your health a } \\
\text { priority. } \\
\text { How to choose plant-based when eating out? See } \\
\text { these tips! } \\
\text { How likely are you to focus on eating plant-based } \\
\text { protein next week? } \\
\text { Text back } 1 \text { (not at all), } 2 \text { (maybe), or } 3 \text { (likely) }\end{array}$ & $\begin{array}{l}\text { Some animals (especially cows) produce large } \\
\text { amounts of a harmful greenhouse gas, methane, by } \\
\text { their gut bacteria. High levels of methane contribute } \\
\text { to global warming! Need help taking the first step? } \\
\text { Tips > } \\
\text { Enjoy Vegetarian Meals I MyPlate } \\
1 \text { in } 4 \text { Americans ate more plant-based protein last } \\
\text { year. You could do the same! Let's join in. What're you } \\
\text { waiting for? It's about time to make our planet a } \\
\text { priority. } \\
\text { How to choose plant-based when eating out? See } \\
\text { these tips! } \\
\text { How likely are you to focus on eating plant-based } \\
\text { protein next week? } \\
\text { Text back } 1 \text { (not at all), } 2 \text { (maybe), or } 3 \text { (likely) }\end{array}$ \\
\hline 7 & $\begin{array}{l}\text { Are you aware that many plant foods are a good } \\
\text { source of protein too? Beans, chickpeas, split peas, } \\
\text { edamame, lentils, quinoa, soybeans, tofu, and tempeh } \\
\text { are some examples of lean protein. Try a simple } \\
\text { protein swap for your next meal! } \\
\text { PS: Lean plant protein is better for your health. } \\
\text { Looking for simple plant-based swaps when cooking? } \\
\text { Check these ideas out! } \\
\text { Did you know legumes (beans and peas) are } \\
\text { considered both vegetable and protein food? You only } \\
\text { need } 1 \frac{1}{2} \text { cup a week for a } 2000 \text { daily calorie intake. It's } \\
\text { a great way to eat both veggies and plant protein! You } \\
\text { already know consuming lean protein and more } \\
\text { veggies is good for your health. } \\
\text { Looking for more plant-based recipes? Explore these } \\
\text { resources from the } \\
\text { Recipes I MyPlate }\end{array}$ & $\begin{array}{l}\text { Are you aware that many plant foods are a good } \\
\text { source of protein too? Beans, chickpeas, split peas, } \\
\text { edamame, lentils, quinoa, soybeans, tofu, and tempeh } \\
\text { are some examples of lean protein. Try a simple } \\
\text { protein swap for your next meal! } \\
\text { PS: Lean plant protein is better for our environment. } \\
\text { Looking for simple plant-based swaps when cooking? } \\
\text { Check these ideas out! } \\
\text { Did you know legumes (beans and peas) are } \\
\text { considered both vegetable and protein food? You only } \\
\text { need } 1 \frac{1}{2} \text { cup a week for a } 2000 \text { daily calorie intake. It's } \\
\text { a great way to eat both veggies and plant protein! You } \\
\text { already know consuming lean protein and more } \\
\text { veggies is good for our planet. } \\
\text { Looking for more plant-based recipes? Explore these } \\
\text { resources from the Recipes I MyPlate }\end{array}$ \\
\hline 8 & $\begin{array}{l}\text { Did you know } 56 \% \text { of people say they try to eat } \\
\text { healthy? You can do the same by choosing plant } \\
\text { proteins to improve your health. Your friends and } \\
\text { family want you to be healthy too! } \\
\text { How to choose plant-based when eating out? See } \\
\text { these tips! } \\
\text { Eat a little more plant-based food ... a little less } \\
\text { animal-based food ... You can make small changes to } \\
\text { your diet and have a big impact on your health! Eating } \\
\text { plant-based doesn't mean giving up meat or dairy, but } \\
\text { just choosing plants in higher proportions. } \\
\text { Looking for simple plant-based swaps when cooking? } \\
\text { Check these ideas out! } \\
\text { How likely are you to focus on eating more fruits and } \\
\text { vegetables next week? } \\
\text { Text back } 1 \text { (not at all), } 2 \text { (maybe), or } 3 \text { (likely) }\end{array}$ & $\begin{array}{l}\text { Did you know } 54 \% \text { of people say they try to eat in an } \\
\text { environmentally sustainability way? You can do the } \\
\text { same by choosing plant proteins to reduce your } \\
\text { environmental footprint. Your friends and family } \\
\text { want you to protect their planet too! } \\
\text { How to choose plant-based when eating out? See } \\
\text { these tips! } \\
\text { Eat a little more plant-based food ... a little less } \\
\text { animal-based food ... You can make small changes to } \\
\text { your diet and have a big impact on the planet! Eating } \\
\text { plant-based doesn't mean giving up meat or dairy, but } \\
\text { just choosing plants in higher proportions. } \\
\text { Looking for simple plant-based swaps when cooking? } \\
\text { Check these ideas out! } \\
\text { How likely are you to focus on eating more fruits and } \\
\quad \text { vegetables next week? } \\
\text { Text back } 1 \text { (not at all), } 2 \text { (maybe), or } 3 \text { (likely) }\end{array}$ \\
\hline
\end{tabular}

\section{References}

1. U.S. Department of Health and Human Services; U.S. Department of Agriculture. 2015-2020 Dietary Guidelines for Americans, 8th ed.; 2015. Available online: https:/ / www.dietaryguidelines.gov/about-dietary-guidelines/previous-editions/2015-dietaryguidelines (accessed on 10 September 2021).

2. U.S. Department of Agriculture; U.S. Department of Health and Human Services. Dietary Guidelines for Americans, 2020-2025, 9th ed. Available online: DietaryGuidelines.gov (accessed on 10 September 2021). 
3. Hoy, K.; Clemens, J.; Moshfegh, A. Estimated Protein Intake from Animal and Plant Foods by U.S. Adults, What We Eat in America, NHANES, 2015-2016. Curr. Dev. Nutr. 2021, 5, 133. [CrossRef]

4. Pasiakos, S.M.; Agarwal, S.; Lieberman, H.R.; Fulgoni, V.L. Sources and Amounts of Animal, Dairy, and Plant Protein Intake of US Adults in 2007-2010. Nutrients 2015, 7, 7058-7069. [CrossRef]

5. Smit, E.; Nieto, F.J.; Crespo, C.J.; Mitchell, P. Estimates of Animal and Plant Protein Intake in US Adults: Results from the Third National Health and Nutrition Examination Survey, 1988-1991. J. Am. Diet. Assoc. 1999, 99, 813-820. [CrossRef]

6. Cotton, P.A.; Subar, A.F.; Friday, J.E.; Cook, A. Dietary sources of nutrients among US adults, 1994 to 1996. J. Am. Diet. Assoc. 2004, 104, 921-930. [CrossRef] [PubMed]

7. Popkin, B.M. Global nutrition dynamics: The world is shifting rapidly toward a diet linked with noncommunicable diseases. Am. J. Clin. Nutr. 2006, 84, 289-298. [CrossRef] [PubMed]

8. Popkin, B.M. Relationship between shifts in food system dynamics and acceleration of the global nutrition transition. Nutr. Rev. 2017, 75, 73-82. [CrossRef]

9. Yamane, T.; Kaneko, S. Is the younger generation a driving force toward achieving the sustainable development goals? Survey experiments. J. Clean. Prod. 2021, 292, 125932. [CrossRef]

10. Powell, P.K.; Durham, J.; Lawler, S. Food Choices of Young Adults in the United States of America: A Scoping Review. Adv. Nutr. 2019, 10, 479-488. [CrossRef] [PubMed]

11. Tuso, P.J.; Ismail, M.H.; Ha, B.P.; Bartolotto, C. Nutritional update for physicians: Plant-based diets. Perm. J. 2013, 17, 61-66. [CrossRef] [PubMed]

12. Hever, J. Plant-Based Diets: A Physician's Guide. Perm. J. 2016, 20, 15-082. [CrossRef]

13. Koeth, R.A.; Wang, Z.; Levison, B.S.; Buffa, J.A.; Org, E.; Sheehy, B.T.; Britt, E.B.; Fu, X.; Wu, Y.; Li, L.; et al. Intestinal microbiota metabolism of l-carnitine, a nutrient in red meat, promotes atherosclerosis. Nat. Med. 2013, 19, 576-585. [CrossRef]

14. Sabaté, J.; Soret, S. Sustainability of plant-based diets: Back to the future. Am. J. Clin. Nutr. 2014, 100, 476S-482S. [CrossRef] [PubMed]

15. McGuire, M. Scientific Report of the 2015 Dietary Guidelines Advisory Committee. Washington, DC: US Departments of Agriculture and Health and Human Services, 2015. Adv. Nutr. 2016, 7, 202-204. [CrossRef] [PubMed]

16. Nelson, M.E.; Hamm, M.W.; Hu, F.B.; Abrams, S.A.; Griffin, T.S. Alignment of Healthy Dietary Patterns and Environmental Sustainability: A Systematic Review. Adv. Nutr. 2016, 7, 1005-1025. [CrossRef]

17. Grummon, A.H.; Goodman, D.; Jaacks, L.M.; Taillie, L.S.; Chauvenet, C.A.; Salvia, M.G.; Rimm, E.B. Awareness of and reactions to health and environmental harms of red meat among parents in the United States. Public Health Nutr. 2021, 1-11. [CrossRef] [PubMed]

18. Neff, R.A.; Edwards, D.; Palmer, A.; Ramsing, R.; Righter, A.; Wolfson, J. Reducing meat consumption in the USA: A nationally representative survey of attitudes and behaviours. Public Health Nutr. 2018, 21, 1835-1844. [CrossRef]

19. Graça, J.; Truninger, M.; Junqueira, L.; Schmidt, L. Consumption orientations may support (or hinder) transitions to more plant-based diets. Appetite 2019, 140, 19-26. [CrossRef]

20. Vainio, A.; Niva, M.; Jallinoja, P.; Latvala, T. From beef to beans: Eating motives and the replacement of animal proteins with plant proteins among Finnish consumers. Appetite 2016, 106, 92-100. [CrossRef]

21. International Food Information Council. 2021 Food \& Health Survey. 2021. Available online: https://foodinsight.org/2021-foodhealth-survey (accessed on 23 October 2021).

22. Leiserowitz, A.; Ballew, M.; Rosenthal, S.; Semaan, J. Climate Change and the American Diet; Yale Program on Climate Change Communication: New Haven, CT, USA, 2020.

23. Pew Research Center. Mobile Fact Sheet. 2021. Available online: https://www.pewresearch.org/internet/fact-sheet/mobile/ (accessed on 5 November 2021).

24. Brown, O.N.; O'Connor, L.E.; Savaiano, D. Mobile MyPlate: A Pilot Study Using Text Messaging to Provide Nutrition Education and Promote Better Dietary Choices in College Students. J. Am. Coll. Health 2014, 62, 320-327. [CrossRef]

25. Santo, K.; Hyun, K.; De Keizer, L.; Thiagalingam, A.; Hillis, G.S.; Chalmers, J.; Redfern, J.; Chow, C.K. The effects of a lifestylefocused text-messaging intervention on adherence to dietary guideline recommendations in patients with coronary heart disease: An analysis of the TEXT ME study. Int. J. Behav. Nutr. Phys. Act. 2018, 15, 45. [CrossRef]

26. O'Brien, L.M.; Palfai, T.P. Efficacy of a brief web-based intervention with and without SMS to enhance healthy eating behaviors among university students. Eat. Behav. 2016, 23, 104-109. [CrossRef]

27. Carfora, V.; Caso, D.; Conner, M. Correlational study and randomised controlled trial for understanding and changing red meat consumption: The role of eating identities. Soc. Sci. Med. 2017, 175, 244-252. [CrossRef]

28. Taufik, D.; Verain, M.C.D.; Bouwman, E.P.; Reinders, M.J. Determinants of real-life behavioural interventions to stimulate more plant-based and less animal-based diets: A systematic review. Trends Food Sci. Technol. 2019, 93, 281-303. [CrossRef]

29. Hekler, E.B.; Gardner, C.D.; Robinson, T.N. Effects of a College Course About Food and Society on Students' Eating Behaviors. Am. J. Prev. Med. 2010, 38, 543-547. [CrossRef] [PubMed]

30. Malan, H.; Challamel, G.A.; Silverstein, D.; Hoffs, C.; Spang, E.; Pace, S.A.; Malagueño, B.L.R.; Gardner, C.D.; Wang, M.C.; Slusser, W.; et al. Impact of a Scalable, Multi-Campus "Foodprint" Seminar on College Students' Dietary Intake and Dietary Carbon Footprint. Nutrients 2020, 12, 2890. [CrossRef] [PubMed] 
31. Larson, N.; Laska, M.N.; Neumark-Sztainer, D. Do young adults value sustainable diet practices? Continuity in values from adolescence to adulthood and linkages to dietary behaviour. Public Health Nutr. 2019, 22, 2598-2608. [CrossRef]

32. Pelletier, J.E.; Laska, M.N.; Neumark-Sztainer, D.; Story, M. Positive Attitudes toward Organic, Local, and Sustainable Foods Are Associated with Higher Dietary Quality among Young Adults. J. Acad. Nutr. Diet. 2013, 113, 127-132. [CrossRef]

33. Webb, T.L.; Joseph, J.; Yardley, L.; Michie, S. Using the Internet to Promote Health Behavior Change: A Systematic Review and Meta-analysis of the Impact of Theoretical Basis, Use of Behavior Change Techniques, and Mode of Delivery on Efficacy. J. Med. Internet Res. 2010, 12, e4. [CrossRef]

34. McClenahan, C.; Shevlin, M.; Adamson, G.; Bennett, C.; O'Neill, B. Testicular self-examination: A test of the health belief model and the theory of planned behaviour. Health Educ. Res. 2007, 22, 272-284. [CrossRef] [PubMed]

35. Gerend, M.A.; Shepherd, J.E. Predicting Human Papillomavirus Vaccine Uptake in Young Adult Women: Comparing the Health Belief Model and Theory of Planned Behavior. Ann. Behav. Med. 2012, 44, 171-180. [CrossRef]

36. Rosenstock, I.M. Historical Origins of the Health Belief Model. Health Educ. Monogr. 1974, 2, 328-335. [CrossRef]

37. Dehdari, T.; Dehdari, L.; Jazayeri, S. Diet-Related Stomach Cancer Behavior among Iranian College Students: A Text Messaging Intervention. Asian Pac. J. Cancer Prev. 2016, 17, 5165-5172. [CrossRef] [PubMed]

38. Hidarnia, A.; Jeihooni, A.K.; Kaveh, M.H.; Hajizadeh, E.; Askari, A. The effect of an educational program based on health belief model on preventing osteoporosis in women. Int. J. Prev. Med. 2015, 6, 115. [CrossRef] [PubMed]

39. Ajzen, I. The Theory of Planned Behavior. Organ. Behav. Hum. Decis. Process. 1991, 50, 179-211. [CrossRef]

40. Wallston, K. Control Beliefs: Health Perspectives. In International Encyclopedia of the Social E Behavioral Sciences; Smelser, N.J., Baltes, P.B., Eds.; Pergamon: Oxford, UK, 2001; pp. 2724-2726. ISBN 978-0-08-043076-8.

41. Dean, M.; Raats, M.M.; Shepherd, R. The Role of Self-Identity, Past Behavior, and Their Interaction in Predicting Intention to Purchase Fresh and Processed Organic Food. J. Appl. Soc. Psychol. 2011, 42, 669-688. [CrossRef]

42. O'Connor, E.L.; Sims, L.; White, K.M. Ethical food choices: Examining people's Fair Trade purchasing decisions. Food Qual. Prefer. 2017, 60, 105-112. [CrossRef]

43. Dowd, K.; Burke, A.K.J. The influence of ethical values and food choice motivations on intentions to purchase sustainably sourced foods. Appetite 2013, 69, 137-144. [CrossRef] [PubMed]

44. Vermeir, I.; Verbeke, W. Sustainable food consumption among young adults in Belgium: Theory of planned behaviour and the role of confidence and values. Ecol. Econ. 2008, 64, 542-553. [CrossRef]

45. Bratanova, B.; Vauclair, C.-M.; Kervyn, N.; Schumann, S.; Wood, R.; Klein, O. Savouring morality. Moral satisfaction renders food of ethical origin subjectively tastier. Appetite 2015, 91, 137-149. [CrossRef]

46. Sparkman, G.; Walton, G.M. Dynamic Norms Promote Sustainable Behavior, Even if It Is Counternormative. Psychol. Sci. 2017, 28, 1663-1674. [CrossRef]

47. Lindsey, L.L.M. The influence of persuasive messages on healthy eating habits: A test of the theory of reasoned action when attitudes and subjective norm are targeted for change. J. Appl. Biobehav. Res. 2017, 22, e12106. [CrossRef]

48. Asril, N.M.; Tabuchi, K.; Tsunematsu, M.; Kobayashi, T.; Kakehashi, M. Predicting Healthy Lifestyle Behaviours among Patients with Type 2 Diabetes in Rural Bali, Indonesia. Clin. Med. Insights Endocrinol. Diabetes 2020, 13, 1-13. [CrossRef]

49. U.S. Department of Agriculture, Agricultural Research Service. Nutrient Intakes from Food and Beverages: Mean Amounts Consumed per Individual, by Gender and Age. 2020, What We Eat in America, NHANES 2017-2019. Available online: www.ars.usda.gov/nea/bhnrc/fsrg (accessed on 20 October 2021).

50. Park, Y.; Dodd, K.W.; Kipnis, V.; Thompson, F.E.; Potischman, N.; Schoeller, D.A.; Baer, D.J.; Midthune, D.; Troiano, R.; Bowles, H.; et al. Comparison of self-reported dietary intakes from the Automated Self-Administered 24-h recall, 4-d food records, and food-frequency questionnaires against recovery biomarkers. Am. J. Clin. Nutr. 2018, 107, 80-93. [CrossRef] [PubMed]

51. Subar, A.F.; Potischman, N.; Dodd, K.W.; Thompson, F.E.; Baer, D.J.; Schoeller, D.A.; Midthune, D.; Kipnis, V.; Kirkpatrick, S.I.; Mittl, B.; et al. Performance and Feasibility of Recalls Completed Using the Automated Self-Administered 24-Hour Dietary Assessment Tool in Relation to Other Self-Report Tools and Biomarkers in the Interactive Diet and Activity Tracking in AARP (IDATA) Study. J. Acad. Nutr. Diet. 2020, 120, 1805-1820. [CrossRef] [PubMed]

52. Plotnikoff, R.C.; Lippke, S.; Trinh, L.; Courneya, K.S.; Birkett, N.; Sigal, R.J. Protection motivation theory and the prediction of physical activity among adults with type 1 or type 2 diabetes in a large population sample. Br. J. Health Psychol. 2010, 15, 643-661. [CrossRef] [PubMed]

53. Haws, K.L.; Winterich, K.P.; Naylor, R.W. Seeing the world through GREEN-tinted glasses: Green consumption values and responses to environmentally friendly products. J. Consum. Psychol. 2014, 24, 336-354. [CrossRef]

54. Hair, J.F.J.; Black, W.C.; Babin, B.J.; Anderson, R.E. Multivariate Data Analysis, 7th ed.; Pearson Education Limited: London, UK, 2013; ISBN 978-1-292-02190-4.

55. Sekaran, U.; Bougie, R. Research Methods for Business: A Skill Building Approach, 8th ed.; Wiley: Hoboken, NJ, USA, 2019; ISBN 978-1-119-56124-8.

56. Bock, B.C.; Heron, K.E.; Jennings, E.G.; Magee, J.C.; Morrow, K.M. User Preferences for a Text Message-Based Smoking Cessation Intervention. Health Educ. Behav. 2012, 40, 152-159. [CrossRef]

57. Cole, D.A.; Maxwell, S.E. Testing Mediational Models with Longitudinal Data: Questions and Tips in the Use of Structural Equation Modeling. J. Abnorm. Psychol. 2003, 112, 558-577. [CrossRef] [PubMed] 
58. Schwartz, S.H. Are There Universal Aspects in the Structure and Contents of Human Values? J. Soc. Issues 1994, 50, 19-45. [CrossRef]

59. Onwezen, M.C.; Bouwman, E.P.; Reinders, M.J.; Dagevos, H. A systematic review on consumer acceptance of alternative proteins: Pulses, algae, insects, plant-based meat alternatives, and cultured meat. Appetite 2021, 159, 105058. [CrossRef]

60. Jaeger, S.R.; Chheang, S.L.; Prescott, J. Variations in the Strength of Association between Food Neophobia and Food and Beverage Acceptability: A Data-Driven Exploratory Study of an Arousal Hypothesis. Nutrients 2021, 13, 3657. [CrossRef]

61. Hoek, A.C.; Pearson, D.; James, S.W.; Lawrence, M.A.; Friel, S. Healthy and environmentally sustainable food choices: Consumer responses to point-of-purchase actions. Food Qual. Prefer. 2017, 58, 94-106. [CrossRef]

62. Gallup Inc. In U.S., More Say Animals Should Have Same Rights as People. Available online: https://news.gallup.com/poll/18 3275/say-animals-rights-people.aspx (accessed on 15 November 2021).

63. Van Loo, E.J.; Hoefkens, C.; Verbeke, W. Healthy, sustainable and plant-based eating: Perceived (mis)match and involvementbased consumer segments as targets for future policy. Food Policy 2017, 69, 46-57. [CrossRef]

64. Graham, D.J.; Pelletier, J.E.; Neumark-Sztainer, D.; Lust, K.; Laska, M.N. Perceived Social Ecological Factors Associated with Fruit and Vegetable Purchasing, Preparation, and Consumption among Young Adults. J. Acad. Nutr. Diet. 2013, 113, 1366-1374. [CrossRef] [PubMed]

65. Munt, A.E.; Partridge, S.R.; Allman-Farinelli, M. The barriers and enablers of healthy eating among young adults: A missing piece of the obesity puzzle: A scoping review. Obes. Rev. 2017, 18, 1-17. [CrossRef]

66. Hall, A.K.; Cole-Lewis, H.; Bernhardt, J.M. Mobile Text Messaging for Health: A Systematic Review of Reviews. Annu. Rev. Public Health 2015, 36, 393-415. [CrossRef] [PubMed]

67. Cole-Lewis, H.; Kershaw, T. Text Messaging as a Tool for Behavior Change in Disease Prevention and Management. Epidemiol. Rev. 2010, 32, 56-69. [CrossRef]

68. Pollard, C.M.; Howat, P.A.; Pratt, I.S.; Boushey, C.J.; Delp, E.J.; Kerr, D.A.; Buchholz, S.; Pfammatter, A. Preferred Tone of Nutrition Text Messages for Young Adults: Focus Group Testing. JMIR mHealth uHealth 2016, 4, e1. [CrossRef] [PubMed]

69. Henchion, M.; Hayes, M.; Mullen, A.M.; Fenelon, M.; Tiwari, B. Future Protein Supply and Demand: Strategies and Factors Influencing a Sustainable Equilibrium. Foods 2017, 6, 53. [CrossRef]

70. International Food Information Council. 2019 Food \& Health Survey. 2019. Available online: https://foodinsight.org/2019-foodand-health-survey / (accessed on 23 September 2021).

71. Kim, H.; Caulfield, L.E.; Garcia-Larsen, V.; Steffen, L.M.; Coresh, J.; Rebholz, C.M. Plant-Based Diets Are Associated with a Lower Risk of Incident Cardiovascular Disease, Cardiovascular Disease Mortality, and All-Cause Mortality in a General Population of Middle-Aged Adults. J. Am. Hear. Assoc. 2019, 8, e012865. [CrossRef] [PubMed]

72. Myers, J. Exercise and Cardiovascular Health. Circulation 2003, 107, e2-e5. [CrossRef] [PubMed]

73. Ritchie, H.; Roser, M. Environmental Impacts of Food Production. Our World in Data. 2020. Available online: https:// ourworldindata.org/environmental-impacts-of-food (accessed on 23 September 2021).

74. Poore, J.; Nemecek, T. Reducing food's environmental impacts through producers and consumers. Science 2018, 360, 987-992. [CrossRef] [PubMed] 